\title{
Zn nanoparticles irradiated with swift heavy ions at low fluences: Optically-detected shape elongation induced by nonoverlapping ion tracks
}

\author{
H. Amekura,,${ }^{1,}$ N. Ishikawa, ${ }^{2}$ N. Okubo, ${ }^{2}$ M. C. Ridgway, ${ }^{3}$ R. Giulian, ${ }^{3}$ K. Mitsuishi,,${ }^{1}$ Y. Nakayama, ${ }^{1}$ Ch. Buchal,${ }^{4}$ \\ S. Mantl, ${ }^{4}$ and N. Kishimoto ${ }^{1}$ \\ ${ }^{1}$ National Institute for Materials Science (NIMS), 3-13 Sakura, Tsukuba, Ibaraki 305-0003, Japan \\ ${ }^{2}$ Japan Atomic Energy Agency (JAEA), Tokai, Ibaraki, Japan \\ ${ }^{3}$ Department of Electronic Materials Engineering, Research School of Physics and Engineering, Australian National University, \\ Canberra, Australia \\ ${ }^{4}$ Institut fuer Bio- und Nanosysteme (IBNI-IT), Forschungszentrum Juelich GmbH, D-52425, Juelich, Germany
}

(Received 25 May 2010; revised manuscript received 10 February 2011; published 3 May 2011)

\begin{abstract}
Elongation of metal nanoparticles (NPs) embedded in silica $\left(\mathrm{SiO}_{2}\right)$ induced by swift heavy-ion ( $\mathrm{SHI}$ ) irradiation, from spheres to spheroids, has been evaluated mainly by transmission electron microscopy (TEM) at high fluences, where tens to thousands of ion tracks were overlapped each other. It is important to clarify whether the high fluences, i.e., track overlaps, are essential for the elongation. In this study the elongation of metal NPs was evaluated at low fluences by linearly polarized optical absorption spectroscopy. Zn NPs embedded in silica were irradiated with $200-\mathrm{MeV} \mathrm{Xe}^{14+}$ ions with an incident angle of $45^{\circ}$. The fluence ranged from $1.0 \times 10^{11}$ to $5.0 \times 10^{13} \mathrm{Xe} / \mathrm{cm}^{2}$, which corresponds to the track coverage ratio $(\mathrm{CR})$ of 0.050 to 25 by ion tracks. A small but certain dichroism was observed down to $5.0 \times 10^{11} \mathrm{Xe} / \mathrm{cm}^{2}(\mathrm{CR}=0.25)$. The comparison with numerical simulation suggested that the elongation of Zn NPs was induced by nonoverlapping ion tracks. After further irradiation each NP experienced multiple SHI impacts, which resulted in further elongation. TEM observation showed the elongated NPs whose aspect ratio (AR) ranged from 1.2 to 1.7 at $5.0 \times 10^{13} \mathrm{Xe} / \mathrm{cm}^{2}$. Under almost the same irradiation conditions, Co NPs with the same initial mean radius showed more prominent elongation with AR of $\sim 4$ at the same fluence, while the melting point (m.p.) of Co is much higher than that of $\mathrm{Zn}$. Less efficient elongation of $\mathrm{Zn} \mathrm{NPs} \mathrm{while} \mathrm{lower} \mathrm{m.p.} \mathrm{is} \mathrm{discussed.}$
\end{abstract}

DOI: 10.1103/PhysRevB.83.205401

PACS number(s): 78.67.Bf, 61.80.Jh, 61.46.Df, 78.20.Fm

\section{INTRODUCTION}

Metal nanoparticles (NPs) receive much attention due to various fascinating optical, electronic, and magnetic properties which are not observed in their bulk counterparts: ultrafast optical response ${ }^{1}$ and strong electric field enhancement ${ }^{2}$ of the surface plasmon resonance (SPR), single electron transport, ${ }^{3}$ superparamagnetism, ${ }^{4,5}$ finite-size effect on phase transitions in NPs, ${ }^{6}$ and so on. These properties can be tuned by controlling the size, size distribution, shape of the NPs, and the matrix composition.

As for the shape control of the metal NPs, a new approach was discovered in 2003 by D'Orleans et al., who irradiated cobalt (Co) NPs embedded in $\mathrm{SiO}_{2}$ by $200-\mathrm{MeV}^{127}$ I swift heavy ions (SHI). ${ }^{7}$ They observed enlargement of NPs while retaining the spherical shape at low fluences (up to $1 \times 10^{12}$ ions $/ \mathrm{cm}^{2}$ ) and elongation of NPs to nanorods at higher fluences $\left(>1 \times 10^{13}\right.$ ions $\left./ \mathrm{cm}^{2}\right)$. One of the fascinating points is that the elongation is induced along the direction of the SHI beam; i.e., the NPs are elongated in the same direction and the direction is controlled by the SHI beam. Anisotropic properties can be expected for even a single elongated NP. However, if the elongation direction of each NP is not aligned, the anisotropy of each NP is smeared with each other. Ensembles of misaligned elongated NPs show little anisotropy. Thus, the aligned nature of the elongated NPs fabricated by SHI irradiation is attractive.

Since the discovery, this phenomenon has been extensively studied by many groups. ${ }^{8-22}$ Here we summarize early discussion of the elongation mechanism, while any decisive mechanisms have yet to be proposed. D'Orleans $\mathrm{et} \mathrm{al} .{ }^{7}$ pointed out that the lattice temperature of smaller NPs increases to a greater extent based on the thermal spike model. They ascribed the enlargement of NPs to vaporization and the elongation to melting of NPs, respectively. The vaporization was assumed to enhance diffusion of metal species and consequently reaggregation and enlargement of NPs. They speculated that thermal pressure in the molten NPs induced the elongation via creep deformation. On the other hand, Roorda et al. reported the elongation of Au cores of Au (silica) core (shell) particles by irradiation of $30-\mathrm{MeV}$ Se ions and suggested an important role of the $\mathrm{SiO}_{2}$ matrix for the elongation of metal NPs. They ascribed the elongation to in-plane stress induced by the ionhammering effect of the $\mathrm{SiO}_{2}$ matrix. ${ }^{8}$ However, Penninkhof et al. ${ }^{10}$ pointed out that the deformation of $\mathrm{Au}$ cores required an electronic energy loss threshold of $3.3 \mathrm{keV} / \mathrm{nm}$, while the ion-hammering effect of $\mathrm{SiO}_{2}$ required no measurable threshold. ${ }^{23,24}$ Klaumuenzer concluded that crystalline Co NPs cannot be measurably deformed by ion hammering via known deformation mechanisms ${ }^{20}$ and suggested models including molten NPs.

Awazu et al. carried out detailed thermal spike calculations and explained the elongation of NPs in term of whether both the NPs and $\mathrm{SiO}_{2}$ matrix melt as a result of the thermal spike effect. ${ }^{16}$ Based on the calculation and experimental confirmation, they insisted that the maximum threshold diameter of $\mathrm{Au}$ NPs fell between 10 and $20 \mathrm{~nm}$. However, recently other groups have experimentally shown the elongation of Au NPs as large as $45 \mathrm{~nm}$ in diameter. ${ }^{21,25}$ Awazu et al. did not propose any scenarios from the melting to the elongation, except briefly 
suggesting a possible role of the in-plane stress of the $\mathrm{SiO}_{2}$ matrix. This model was also suggested by Oliver et al. ${ }^{11}$

Ridgway et al. also pointed out ${ }^{19}$ that Co NPs larger than $50 \mathrm{~nm}$ in diameter showed elongation under 196-MeV Au irradiation, while thermal spike calculations predicted that only Co NPs smaller than $20 \mathrm{~nm}$ in diameter should elongate. ${ }^{7}$ Rather, they clearly showed the minimum threshold size of NPs for elongation ${ }^{12,15}$ and correlated it with the diameter of the ion track.

Another important observation was reported by Dawi et al., ${ }^{21}$ who evaluated the effect of the particle density on the elongation behavior. They observed that the elongation almost ceases at high fluence when the particle density is low, while the elongation continues when the particle density is high. This fact indicates that the mass transport between NPs plays an important role in the elongation process, at least at high fluence. The elongation phenomenon may not be a simple consequence of melting due to the thermal spike but rather a more complicated dynamical process, including mass transport between NPs.

A new finding has been obtained from nonmetallic $\mathrm{Ge}$ NPs irradiated by SHIs. Whereas free-standing amorphous or amorphizable nonmetallic NPs show oblate deformation only, Ge NPs embedded in $\mathrm{SiO}_{2}$ showed either prolate or oblate deformation depending on the NP size. ${ }^{22}$

While the importance of the melting has been pointed out by several authors, few scenarios from melting to elongation have been proposed, except creep deformation ${ }^{7}$ or (molten) matter flow in the ion track ${ }^{20}$ induced by thermal pressure of the NPs and anisotropic deformation of the $\mathrm{SiO}_{2}$ matrix. ${ }^{8}$ Furthermore, new unexpected behaviors have been reported; i.e., particle density dependence of Au NPs and bimode deformation of Ge NPs. It seems that we are still far away from not only clarification of the mechanism but also a complete description of the phenomenon.

Until now, studies have been carried out mainly on a limited species of NPs such as $\mathrm{Au}, \mathrm{Ag}, \mathrm{Cu}, \mathrm{Pt}$, and Ge. However, a breakthrough could be obtained from unexplored metal species. This paper describes the study of the elongation phenomenon of Zn NPs, which have rarely been studied. The melting points of bulk $\mathrm{Zn}$ and of $\mathrm{Zn}$ NPs are $419.6^{\circ} \mathrm{C}$ and $250^{\circ}-420^{\circ} \mathrm{C}$, respectively, ${ }^{26,27}$ much lower than the bulk melting points of bulk $\mathrm{Au}, \mathrm{Ag}$, and $\mathrm{Cu}\left(1064.4^{\circ}, 961.9^{\circ}\right.$, and $1083.4^{\circ} \mathrm{C}$ ). Since the melting of NPs is considered to play an important role for the elongation, the much lower melting point of $\mathrm{Zn}$ NPs could induce notable differences. While the noble metal NPs show strong and sharp SPR, Zn NPs show a broad absorption in the ultraviolet (UV) region and a sharp but very weak peak in the near infrared (NIR) region. ${ }^{28}$ Differences in optical behavior from the noble metal NPs can be observed.

A question arising for the elongation process is whether overlaps of ion tracks are essential for the elongation of NPs. It is well known that the distinguishable elongation is induced at the fluence of $\sim 1 \times 10^{14}$ ions $/ \mathrm{cm}^{2}$ or higher, where one NP is subjected to impacts of $\sim 100$ SHIs or more. Furthermore, the elongation was observed by relatively low energy ions of $8 \mathrm{MeV} \mathrm{Si}$, if very high fluence of $10^{16}$ ions $/ \mathrm{cm}^{2}$ was applied. ${ }^{11}$ It is an important issue to understand whether the multiple collisions with SHIs are essential for the elongation or simply the elongation induced by a single impact is so small that
TABLE I. Energy losses and projected range of 200-MeV Xe ions in amorphous $\mathrm{SiO}_{2}$ calculated by SRIM2008. ${ }^{32}$

Electronic energy loss, $S_{e}$

Nuclear energy loss, $S_{n}$

$14.5 \mathrm{keV} / \mathrm{nm}$

Projected range, $R_{p}$

$\mathrm{nm}$

$21.7 \mu \mathrm{m}$

integration is necessary. In this paper, this point is discussed using optical spectroscopy.

\section{EXPERIMENTAL}

$\mathrm{Zn}$ NPs were fabricated in silica glass of KU-1 type $(\mathrm{OH} \sim 820 \mathrm{ppm})$ by implantation of $\mathrm{Zn}$ ions at $60 \mathrm{keV}$ to a fluence of $1.0 \times 10^{17} \mathrm{ions} / \mathrm{cm}^{2}$. Detailed fabrication conditions and fundamental properties of the NPs are described in our previous papers. ${ }^{26-31}$ The silica samples containing Zn NPs were irradiated with $200 \mathrm{MeV} \mathrm{Xe}^{14+}$ ions at room temperature (RT) using the tandem accelerator at the Japan Atomic Energy Agency, Tokai Research and Development Center (JAEA-Tokai). The electronic and nuclear energy losses, and the projected range of $200 \mathrm{MeV}$ Xe ion in $\mathrm{SiO}_{2}$, were estimated from SRIM2008 code ${ }^{32}$ and are summarized in Table I. For comparison, silica samples without Zn NPs were also irradiated under the same conditions.

The SHI fluence ranged from $1.0 \times 10^{11}$ to $5.0 \times 10^{13}$ $\mathrm{Xe} / \mathrm{cm}^{2}$, with an ion flux of $\sim 0.3$ or $\sim 4$ particle-nA $/ \mathrm{cm}^{2}$. Samples were irradiated with an incident angle of $45^{\circ}$ from the surface normal given this configuration is appropriate for the detection of NP elongation using linearly polarized-light absorption spectroscopy. If the sample was irradiated with an incident angle of $0^{\circ}$, the NPs would be elongated in the direction of the surface normal as shown schematically in Fig. 1. Such elongated NPs would appear isotropic in the plane

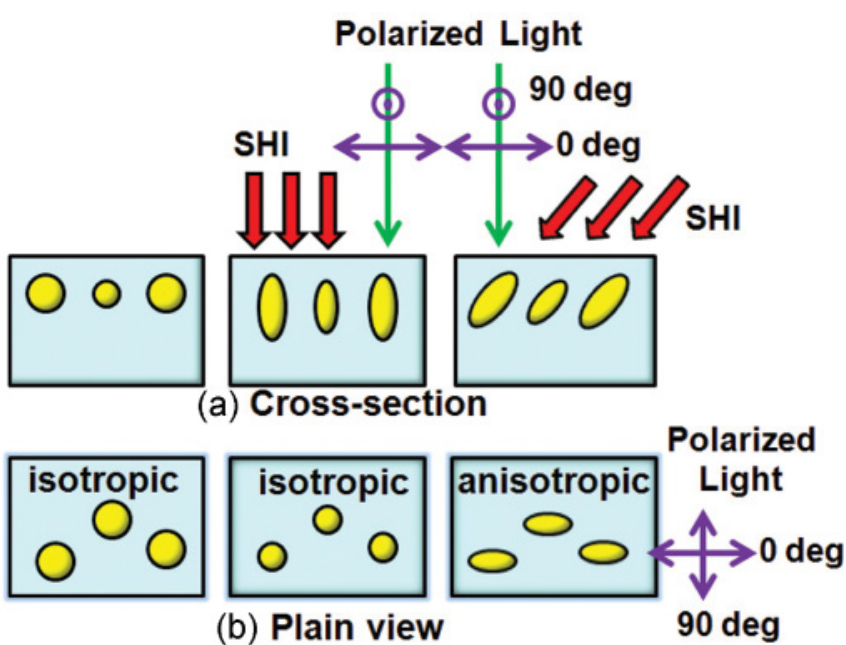

\section{unirrad normal-irrad inclined-irrad}

FIG. 1. (Color online) Schematically depicted configuration of the elongation of NPs induced by normal and inclined irradiations, shown in (a) cross-sectional and (b) plain views. Anisotropy in the plain view is induced only by the inclined irradiation but not by the normal irradiation. The definition of the angle of light polarization is also shown. 
of the sample surface, inhibiting detection of the anisotropy by the absorption spectroscopy. If the sample was irradiated with SHIs with an inclined direction, the anisotropy could be observed in the surface plane, i.e., the elongation could be detected by absorption spectroscopy.

An important quantity is the coverage ratio (CR) of ion tracks over the irradiated layer. Kluth et al. ${ }^{17}$ have determined the ion-track radius $r$ of $\mathrm{Xe}$ and $\mathrm{Au}$ ions in amorphous $\mathrm{SiO}_{2}$ as a function of electronic energy loss $S_{e}$ by small-angle x-ray scattering (SAXS). In our case of $200 \mathrm{MeV} \mathrm{Xe}$ in $\mathrm{SiO}_{2}$, the electronic energy loss $S_{e}$ is $14.5 \mathrm{keV} / \mathrm{nm}$ (Table I), which corresponds to an ion track radius $r$ of $\sim 4 \mathrm{~nm}$. The CR is defined as

$$
\mathrm{CR}=\pi r^{2} \Phi
$$

where $\Phi$ denotes the fluence. The CR indicates how many times on average an area of sample surface is intersected by an ion track, which ranged from 0.050 to 25 in this study. It is known that multiple impacts of SHIs are necessary for significant elongation of NPs and thus the quantity CR is a useful measure.

A dual beam spectrometer was used in the optical transmission measurements in the wavelength region of 215-1700 nm with a resolution of $1 \mathrm{~nm}$ at RT. An optical polarizer (extinction ratio $<5 \times 10^{-5}$ in this wavelength region) was inserted in front of the sample. The results were shown in the form of optical density $\left(-\log _{10} T\right)$ without correction for reflection, where $T$ denotes the transmittance.

Cross-sectional transmission electron microscopy (XTEM) was carried out at an acceleration voltage of $200 \mathrm{kV}$. Grazing incidence $\mathrm{x}$-ray diffraction (GIXRD) with a fixed incident angle of $3^{\circ}$ was performed using a $\mathrm{Cr} K \alpha$ line. The diffracted $\mathrm{x}$ ray was detected by a position-sensitive proportional counter. Rutherford backscattering spectrometry (RBS) was used to determine the depth profiles and total content of $\mathrm{Zn}$ atoms in the sample using a $2.06 \mathrm{MeV} \mathrm{He}^{+}$beam of $1 \mathrm{~mm}$ in diameter with a scattering angle of $160^{\circ}$. The data were analyzed with the RUMP code. ${ }^{33}$ Reflection spectra in the infrared (IR) region (350-4000 $\mathrm{cm}^{-1}$ ) were measured at RT using a single-beam Fourier-transform spectrometer with a $4 \mathrm{~cm}^{-1}$ resolution and an incident angle of $10^{\circ}$ from the surface normal.

\section{RESULTS}

Before discussing the elongation, irradiation effects of the SHIs on the metal content, the crystallinity of the NPs, and $\mathrm{SiO}_{2}$ matrix are first described. Although some reports have been already given on the SHI irradiation effects on the content and the crystallinity of metal NPs, Zn NPs could be a special case because the melting point is $<420^{\circ} \mathrm{C}$ much lower than those of metal species which showed the elongation. It was important to confirm the basic properties, because these procedures provided a basis to discuss the elongation phenomenon. It should be also noted that the effects on the $\mathrm{SiO}_{2}$ matrix were well described using the ion-track radius determined by Kluth et al., ${ }^{17}$ which guarantees the validity of this radius value in the discussion of the elongation.

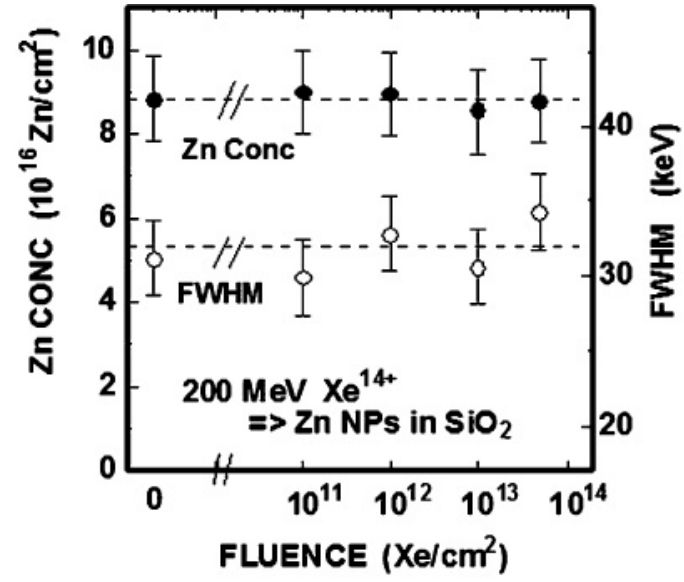

FIG. 2. Fluence dependence of $\mathrm{Zn}$ areal concentration (solid circles) and of FWHM of $\mathrm{Zn}$ peak (open circles) determined from the RBS spectra of Zn NPs embedded in silica irradiated with $200-\mathrm{MeV}$ $\mathrm{Xe}^{14+}$ ions.

\section{A. Irradiation effects on metal content, crystallinity, and $\mathrm{SiO}_{2}$ matrix}

RBS spectra of samples in unirradiated state and after irradiations of $200 \mathrm{MeV} \mathrm{Xe} \mathrm{Xe}^{14+}$ were measured. The areal density of $\mathrm{Zn}$ atoms was determined and is shown in Fig. 2 by solid circles. The $\mathrm{Zn}$ content was constant within the experimental uncertainty even up to the highest fluence of $5.0 \times 10^{13} \mathrm{Xe} / \mathrm{cm}^{2}$. This contrasts with the work of Joseph et al. ${ }^{34}$ who irradiated Au NPs in silica glass with $100 \mathrm{MeV}$ $\mathrm{Au}^{8+}$ ions at $100 \mathrm{~K}$ up to $1 \times 10^{14} \mathrm{ions} / \mathrm{cm}^{2}$ and observed enhanced sputtering and drastic loss of Au NPs. The full width at half maximum (FWHM) of the $\mathrm{Zn}$ peak was also plotted, as open circles, as a function of fluence in Fig. 2. While the FWHM slightly increased after irradiation of $5.0 \times 10^{13} \mathrm{Xe} / \mathrm{cm}^{2}$, this change was minimal and within the experimental uncertainty.

GIXRD petterns are shown in Fig. 3 and the (101) diffraction peak from crystalline Zn NPs is clearly observed. Even with increasing fluence, the peak intensity does not decrease, but the peak width becomes slightly narrower, most likely as a result of NP enlargement. The experimental results indicate that $\mathrm{Zn}$ NPs remain in a hexagonal crystalline phase even up to the highest fluence of $5.0 \times 10^{13} \mathrm{Xe} / \mathrm{cm}^{2}$. This contrasts with irradiation in the nuclear stopping regime where amorphization of metal NPs by 5-MeV Sn ion irradiation was reported for $\mathrm{Cu} \mathrm{NPs}{ }^{35}$ and $\mathrm{Co} \mathrm{NPs}^{36}$ embedded in $\mathrm{SiO}_{2}$.

More drastic change was induced on a strong and broad peak around $2 \theta \sim 31^{\circ}$, which is ascribed to amorphous $\mathrm{SiO}_{2}$. The peak intensity gradually decreased up to a fluence of $1.0 \times 10^{12} \mathrm{Xe} / \mathrm{cm}^{2}$ and then drastically decreased as shown in Fig. 4(b). According to past studies on $\mathrm{SiO}_{2}$ irradiated with $\mathrm{keV}$ to several $\mathrm{MeV}$ ions, ${ }^{37}$ irradiation broadens the bond angle distribution of the $\mathrm{SiO}_{2}$ network, as if the network was heated to a few thousand degrees of fictive temperature and then rapidly quenched to RT. This broadening of the bond-angle distribution yields the broadening and decrease in intensity apparent in the XRD peak of the amorphous $\mathrm{SiO}_{2}$.

The radiation-induced bond-angle broadening can be also monitored via the vibrational frequency shifts of the $\mathrm{SiO}_{2}$ 


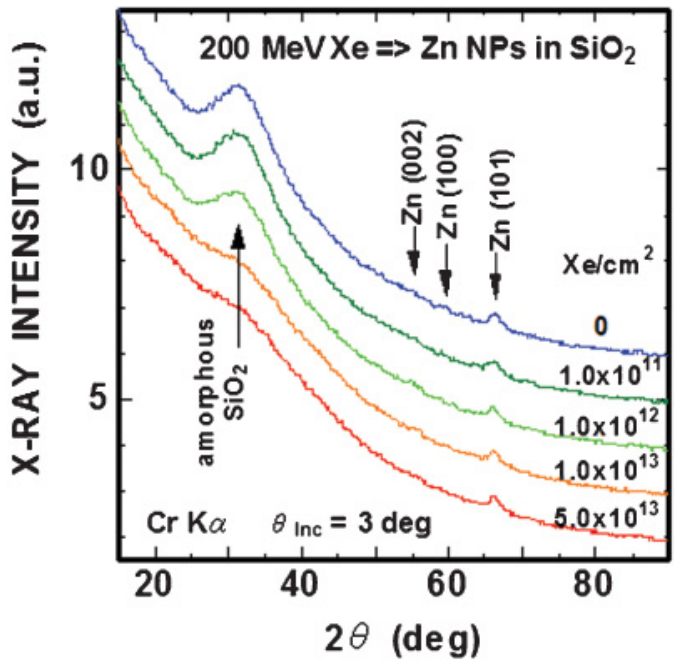

FIG. 3. (Color online) Glancing incident $x$-ray diffraction (GIXRD) patterns from $\mathrm{Zn}$ NPs in silica in unirradiated state and after irradiation of $200-\mathrm{MeV} \mathrm{Xe} \mathrm{e}^{14+}$ ions at four different fluences. The samples were irradiated by the $\mathrm{Cr} \mathrm{K} \alpha$ x-ray with a fixed incident angle of $3^{\circ}$. The patterns are vertically shifted with each other for clarity. The diffraction angles of $\mathrm{Zn}$ (101), (100), (002), and amorphous $\mathrm{SiO}_{2}$ are indicated by arrows.

matrix. ${ }^{37}$ Figure 4(a) shows optical reflectance spectra in the IR region. Three vibrational modes of $\mathrm{SiO}_{2}$, i.e., the $\omega_{1}, \omega_{3}$, and $\omega_{4}$ modes, are observed. Ion irradiation induces shifts of the $\omega_{1}, \omega_{3}$, and $\omega_{4}$ modes to lower, higher, and lower wave numbers, respectively. The peak frequency of the $\omega_{4}$ mode is plotted against the fluence using solid circles in Fig. 4(b). Transformation from the unirradiated state to the well-irradiated state is clearly observed.

The structural changes induced in the $\mathrm{SiO}_{2}$ matrix inferred from the reduction of the XRD peak of the $\mathrm{SiO}_{2}$ matrix (Fig. 3) and shifts of the vibration frequencies of the $\mathrm{SiO}_{2}$ matrix [Fig. 4(a)] show a similar fluence dependence initially then saturate by $1 \times 10^{13} \mathrm{Xe} / \mathrm{cm}^{2}$ as shown in Fig. 4(b). These fluence dependences are reproduced as shown in dotted lines in Fig. 4(b), with presuming that a region covered by ion tracks transforms to the saturated damaged state without any recovery. Moreover, overlaps of the tracks do not induce further changes. The area hit by ions once or more is described as

$$
A_{\text {irrad }}(\Phi)=A_{o}\{1-\exp (-\sigma \Phi)\},
$$

where $\sigma=\pi r^{2}$ and $A_{o}$ the initial unirradiated area. Consequently, the fluence dependence of quantity $Q\left(\Phi_{n}\right)$ is described as

$$
\begin{aligned}
Q(\Phi) & =Q(\infty) \frac{A_{\text {irrad }}(\Phi)}{A_{o}}+Q(0) \frac{A_{o}-A_{\text {irrad }}(\Phi)}{A_{o}} \\
& =\{Q(0)-Q(\infty)\} \exp (-\sigma \Phi)+Q(\infty) .
\end{aligned}
$$

As shown in Fig. 4(b), the data points were well reproduced using the track radius of $4 \mathrm{~nm}$, which was reported by Kluth et al. ${ }^{17}$ These data confirm the reliability of the track-radius value, and this value is used for discussion of the elongation later.
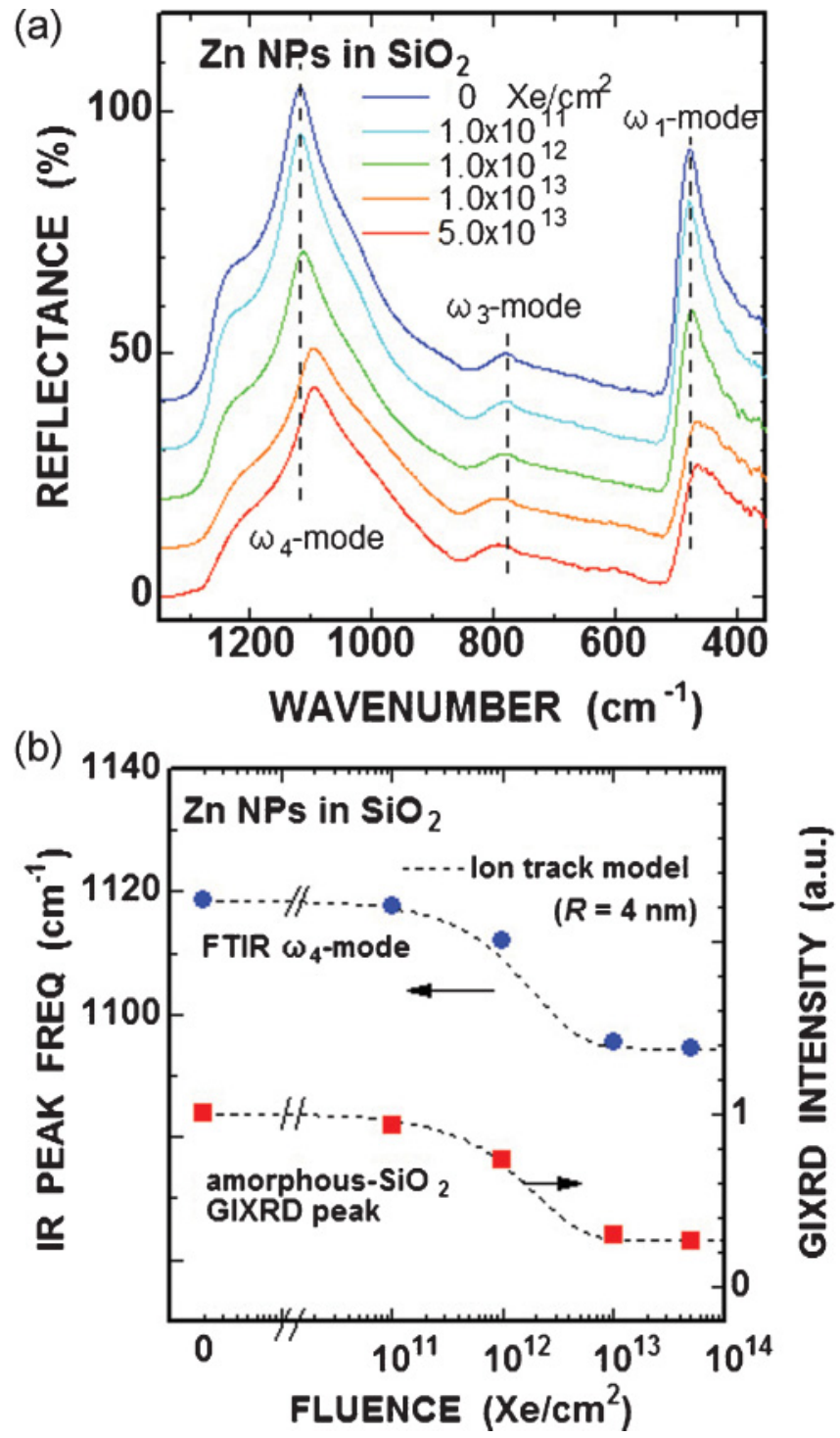

FIG. 4. (Color online) (a) Fourier transform infrared spectroscopy reflection spectra from $\mathrm{Zn}$ NPs in silica in unirradiated state and after irradiation of $200 \mathrm{MeV} \mathrm{Xe}^{14+}$ ions at four different fluences. The spectra are vertically shifted with each other for clarity. The vibration frequencies of the $\omega_{1}, \omega_{3}$, and $\omega_{4}$ peaks of the $\mathrm{SiO}_{2}$ matrix in the unirradiated state are indicated by broken lines. (b) Fluence dependences of the frequency of the $\omega_{4}$ peak and of the diffraction intensity of the amorphous $\mathrm{SiO}_{2}$ peak in the GIXRD pattern shown in Fig. 3 are plotted by circles and squares, respectively. Broken lines indicate the dependences calculated from the track coverage model described by Eq. (3).

\section{B. Elongation of NPs}

Figures 5(a) and 5(b) show optical density spectra of the $\mathrm{Zn}$ NPs in silica against linearly polarized light. The spectra were recorded at polarization angles of $0^{\circ}, 45^{\circ}$, and $90^{\circ}$, where the definition of the angle is shown schematically in Fig. 1. Figure 5(a) shows changes of the ultraviolet (UV) peak around $4.8 \mathrm{eV}$ induced by the SHI irradiation. In the unirradiated state, the spectra recorded at three different polarizations coincided with each other, indicating isotropic shapes of Zn NPs, i.e., spheres. Even after irradiation up to $1.0 \times 10^{11} \mathrm{Xe} / \mathrm{cm}^{2}(\mathrm{CR}=$ 

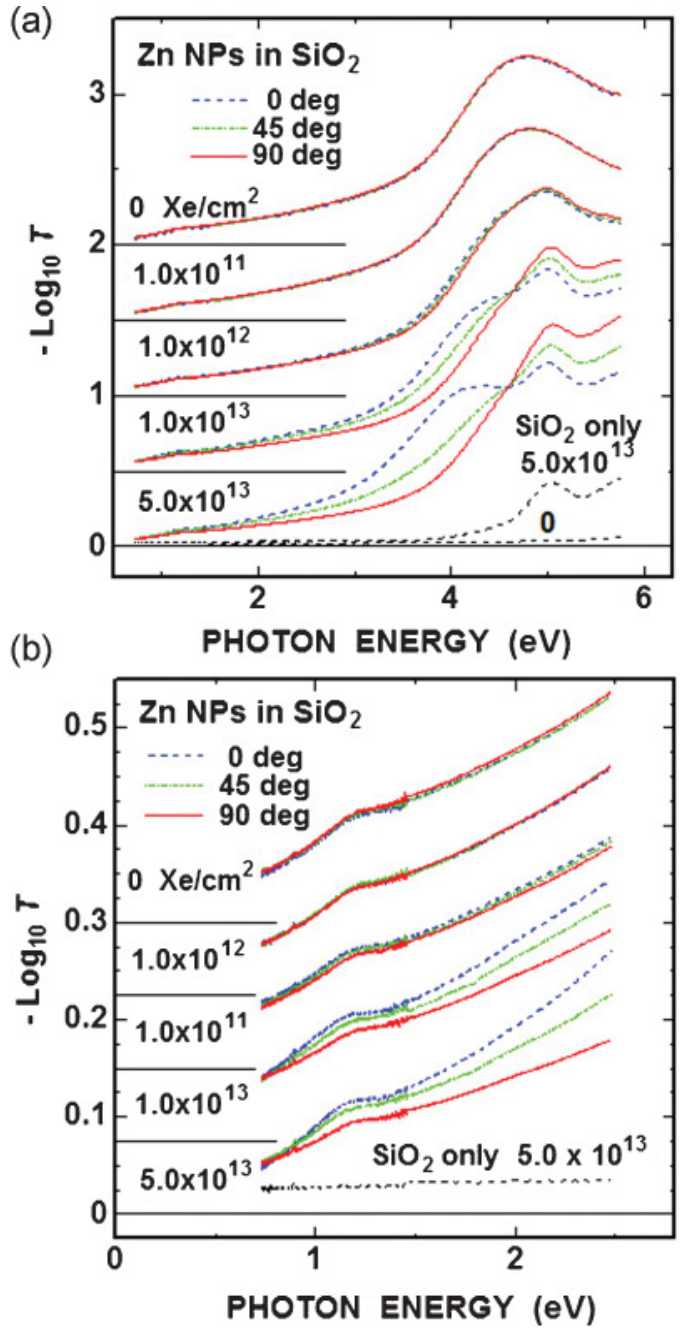

FIG. 5. (Color online) Optical-density spectra of Zn NPs in silica in unirradiated state and after irradiation of $200 \mathrm{MeV} \mathrm{Xe}^{14+}$ ions at four different fluences, (a) whole the spectra and (b) magnified spectra around the NIR peak. Linearly polarized light with the polarization of $0^{\circ}, 45^{\circ}$, and $90^{\circ}$ was used. The spectra are vertically shifted with each other for clarity and the horizontal lines indicate the baselines. For references, the spectra of silica glass without $\mathrm{Zn} \mathrm{NPs} \mathrm{(denoted} \mathrm{as}$ " $\mathrm{SiO}_{2}$ only") in unirradiated state and after the irradiation of $5.0 \times$ $10^{13} \mathrm{Xe} / \mathrm{cm}^{2}$ are also shown.

0.050), the three spectra remained coincident with each other. However, after irradiation of $1.0 \times 10^{12} \mathrm{Xe} / \mathrm{cm}^{2}(\mathrm{CR}=0.50)$, a small but measurable deviation was observed between the different polarizations; i.e., the spectrum recorded at $0^{\circ}\left(90^{\circ}\right)$ shifted to lower (higher) energy side. Furthermore, a new structure appeared at $\sim 5 \mathrm{eV}$. With increasing fluence, the deviation between the different polarizations, i.e., dichroism, became more pronounced up to $5.0 \times 10^{13} \mathrm{Xe} / \mathrm{cm}^{2}(\mathrm{CR}=25)$ and the new structure around $5 \mathrm{eV}$ became more prominent.

For comparison, silica samples without Zn NPs were irradiated under the same conditions and the optical density spectra were measured. Spectra for the unirradiated state and at the fluence of $5.0 \times 10^{13} \mathrm{Xe} / \mathrm{cm}^{2}$ are shown as broken lines in Fig. 5(a). A similar structure was observed around $5 \mathrm{eV}$, indicating radiation damage as the origin. Note that the irradiated silica did not show any polarization dependence and
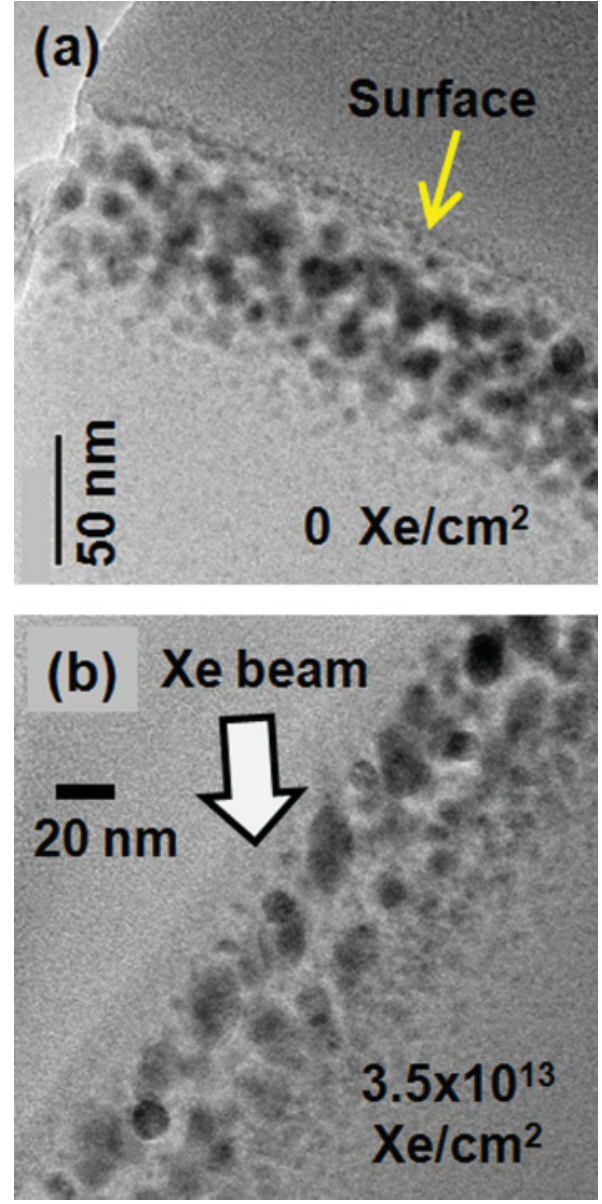

FIG. 6. (Color online) Cross-sectional TEM images of Zn NPs in silica glass in (a) an unirradiated state and (b) after irradiation of $200 \mathrm{MeV} \mathrm{Xe}{ }^{14+}$ ions to a fluence of $5.0 \times 10^{13} \mathrm{Xe} / \mathrm{cm}^{2}(\mathrm{CR}=25)$. As indicated by an arrow in (b), the sample was irradiated with an incident angle of $45^{\circ}$ from the surface normal.

thus the observed dichroism is not due to the $\mathrm{SiO}_{2}$ but to the elongated $\mathrm{Zn} \mathrm{NPs}$, while the peak at $5 \mathrm{eV}$ is ascribed to radiation damage in $\mathrm{SiO}_{2}$.

Direct confirmation of the elongation was obtained by XTEM. Spherically shaped Zn NPs of $10 \pm 2 \mathrm{~nm}$ in diameter were observed before SHI irradiation as shown in Fig. 6(a). After irradiation of $5.0 \times 10^{13} \mathrm{Xe} / \mathrm{cm}^{2}(\mathrm{CR}=25)$, spheroids of $12-26 \mathrm{~nm}$ in major diameter and $10-15 \mathrm{~nm}$ in minor diameter were observed, as shown in Fig. 6(b), to coexist with spherical NPs of $10 \mathrm{~nm}$ or less. Smaller NPs retain the spherical shape as consistent with the existence of the minimum threshold size for elongation reported in the literature. ${ }^{7,15,19}$ It is also clearly apparent that an enlargement of NP volume, compared with the unirradiated state, is induced by SHI irradiation.

Since the polarization-dependent absorption, i.e., dichroism, due to the elongation of $\mathrm{Zn} \mathrm{NPs} \mathrm{was} \mathrm{observed} \mathrm{at}$ $1.0 \times 10^{12} \mathrm{Xe} / \mathrm{cm}^{2}(\mathrm{CR}=0.50)$ but not at $1.0 \times 10^{11} \mathrm{Xe} / \mathrm{cm}^{2}$ $(\mathrm{CR}=0.050)$ as shown in Fig. 5(a), more detailed fluence dependence was evaluated. The absorption difference at $3.8 \mathrm{eV}$ between $0^{\circ}$ and $90^{\circ}$ was plotted in Fig. 7 against the fluence. While the absorption difference decreases with decreasing the fluence, a certain measurable difference was observed down to 


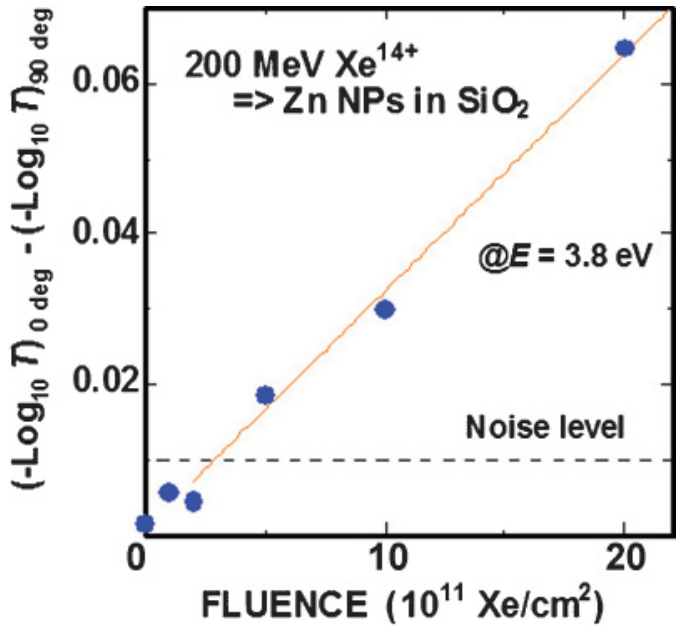

FIG. 7. (Color online) Differences of optical densities at $3.8 \mathrm{eV}$ between the polarization of $0^{\circ}$ and that $90^{\circ}$ are plotted against the fluence. A horizontal broken line indicates experimental uncertainty.

$5.0 \times 10^{11} \mathrm{Xe} / \mathrm{cm}^{2}(\mathrm{CR}=0.25)$. Even at $2.0 \times 10^{11} \mathrm{Xe} / \mathrm{cm}^{2}$ $(\mathrm{CR}=0.10)$, the difference was observed. However, the difference was smaller than experimental uncertainty of the instrument. Consequently, we have observed the elongation of NPs down to $5.0 \times 10^{11} \mathrm{Xe} / \mathrm{cm}^{2}(\mathrm{CR}=0.25)$ but could not at $2.0 \times 10^{11} \mathrm{Xe} / \mathrm{cm}^{2}(\mathrm{CR}=0.10)$. This result does not exclude the existence of the elongation at $2.0 \times 10^{11} \mathrm{Xe} / \mathrm{cm}^{2}$, but simply we could not observed the elongation because the signal was too small for our instrument.

Figure 5(b) shows polarization-dependent optical density spectra of the NIR peak of Zn NPs. Similar with the UV band, no dichroism was observed in the unirradiated state and after irradiation up to $1.0 \times 10^{11} \mathrm{Xe} / \mathrm{cm}^{2}$. After irradiation of $1.0 \times 10^{13} \mathrm{Xe} / \mathrm{cm}^{2}$, the high-energy side of the NIR peak of the $0^{\circ}$ polarization increases due to the low-energy shift of the UV band of the same polarization. Intensity of the NIR peak increases in the $0^{\circ}$ polarization but decreases in the $90^{\circ}$ polarization. These behaviors are semiquantitatively reproduced by calculations described in the Discussion section (not shown).

\section{DISCUSSION}

\section{A. Optical extinction of elongated NPs}

Various properties of Zn NPs and of silica may simultaneously change with the SHI irradiation. An arising question is whether the observed optical changes can be interpreted as a simple consequence of the elongation of Zn NPs only or if other additional irradiation-induced effects should be considered. In order to answer this question, the optical extinction spectra of uniaxially elongated NPs were calculated using Rayleigh theory and were compared with the experimental spectra.

Extinction of light intensity $I$ going through a layer of thickness $z$, which is filled by homogenously- dispersed but uniaxially aligned elongated NPs (spheroids), is described as

$$
I=I_{o} \exp \left(-N C_{\mathrm{ext}} z\right)
$$

where $N, C_{\text {ext }}$, and $I_{o}$ denote the number density of NPs, the extinction cross section, and the incident intensity, respectively. In the quasistatic limit, ${ }^{38} C_{\text {ext }}$ is described by

$$
C_{\text {ext }}=k \operatorname{Im}(\alpha)+\frac{k^{4}}{6 \pi}|\alpha|^{2},
$$

where $k=2 \pi / \lambda$ and $\alpha$ is the polarizability. The first and the second terms describe the absorption and the scattering cross sections, respectively. In our case of nanometric particles, the first term (absorption) was always two or three orders of the magnitude larger than the second term (scattering).

As described later, the dielectric function of Zn NPs was derived from that of bulk $\mathrm{Zn}$ with a modification due to the nanometric size. However, the size dependence is not explicitly included in the calculations. For prolates $(a>b=$ $c$ ) whose major and minor semiaxes are $a$ and $b$, respectively, the eccentricity $e$ and the aspect ratio AR are defined as

$$
e=\sqrt{1-\left(\frac{b}{a}\right)^{2}}
$$

and

$$
\mathrm{AR}=a / b .
$$

The polarizability against the field parallel to the principal axis $i(i=a, b)$ is given by

$$
\alpha_{i}=\left(\frac{4 \pi}{3} a b^{2}\right) \frac{\varepsilon_{\mathrm{NP}}-\varepsilon_{d}}{\varepsilon_{d}+L_{i}\left(\varepsilon_{\mathrm{NP}}-\varepsilon_{d}\right)},
$$

where $\varepsilon_{N P}, \varepsilon_{d}$, and $L_{i}$ denote (complex) dielectric functions of NPs, of matrix, and the geometrical depolarization factors, respectively. The depolarization factor $L_{i}$ is given by

$$
L_{a}=\frac{1-e^{2}}{e^{2}}\left\{\frac{1}{2 e} \ln \left(\frac{1+e}{1-e}\right)-1\right\}
$$

and

$$
L_{b}=\frac{1}{2}\left(1-L_{a}\right) .
$$

Previously, we determined the dielectric function of $\mathrm{Zn}$ from the first-principles band calculations. ${ }^{26}$ The band calculation derives the complex dielectric function of bulk $\mathrm{Zn}$ only. The dielectric function of the NPs was determined in the following way: We assume that the dielectric function is described by a sum of the Drude term and the interband term such as,

$$
\varepsilon_{\text {bulk }}(\omega)=\left\{1-\frac{\omega_{p}^{2}}{\omega^{2}+i(\omega / \tau)}\right\}+\varepsilon_{\text {interband }}(\omega)
$$

and that the dielectric function of the NPs is the same as the bulk one except the value of the relaxation time $\tau$ of the Drude term. In NPs, the mean-free-path of carriers is reduced from the bulk value due to scattering at NP boundary. The value of $\tau$ was determined to reproduce the experimental extinction spectrum of spherical Zn NPs.

The calculated spectra for the polarizations of $0^{\circ}$ and $90^{\circ}$ are shown in Fig. 8(b). Whereas the $90^{\circ}$ polarization excites the transverse mode $(i=b)$ only, the $0^{\circ}$ polarization excites both the longitudinal and the transverse modes $(i=a, b)$, as shown in Fig. 1. In fact, a spectrum of the $0^{\circ}$ polarization at $\mathrm{AR}=$ 1.657 shows a trapezoid-like peak which can be ascribed to the 

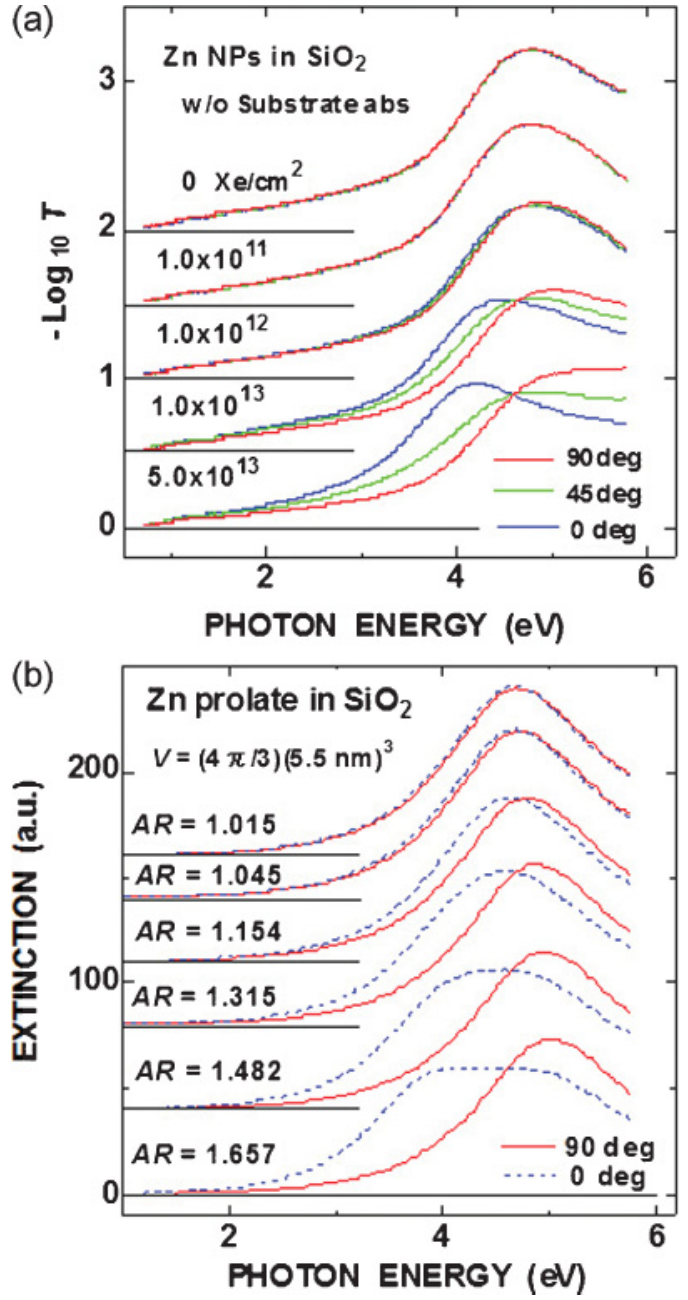

FIG. 8. (Color online) (a) The same optical-density spectra as shown in Fig. 5(a) but the contributions from the silica matrix were subtracted. (b) Calculated optical extinction spectra of a Zn prolate in silica with various ARs. Polarized spectra along the projected major axis $\left(0^{\circ}\right)$ of the prolate and those along one of the minor axes $\left(90^{\circ}\right)$ are shown by broken and solid lines, respectively. While changing the $\mathrm{AR}$, the volume of the prolate is kept constant which corresponds to a sphere of $11 \mathrm{~nm}$ in diameter.

superposition of two Gaussian peaks as shown in Fig. 8(b). For comparison, the experimental spectra are shown in Fig. 8(a), which were subtracted radiation damage of silica matrix.

It should be noted that we have measured the optical transmittance of NP composites while the Rayleigh theory calculates the transmittance of NPs in infinite space. The reflections at the implanted and rear surfaces of the samples are not included in the Rayleigh theory. However, in NP composites, the reflectivities at the surfaces are strongly dependent on the photon energy and may change and become anisotropic with the fluence. Also the irradiation may change the dielectric function of the silica matrix. (However, it should be noted that we did not observe dichroism from silica matrix without $\mathrm{Zn} \mathrm{NPs,} \mathrm{indicating} \mathrm{that} \mathrm{anisotropic}$ changes of the dielectric function of the silica were negligible.) Consequently, the comparison between the experiments and the calculations includes a certain ambiguity. However, the calculations semiquantitatively reproduced the experimental results shown in Fig. 8(a).

The calculation shows that shift of absorption is visible even at the elongation of NP from AR $=1.000$ to 1.015. Although it is not clear whether the high sensitivity of $1.5 \%$ in $\mathrm{AR}$ is attainable in a realistic measurement system, this calculation guarantees high sensitivity of this method.

\section{B. Monte Carlo simulation of track overlap}

We have observed spectroscopic evidence of the elongation of NPs at very low fluence of $5 \times 10^{11} \mathrm{Xe} / \mathrm{cm}^{2}(\mathrm{CR}=0.25)$. If it was at, e.g., $\mathrm{CR} \sim 0.01$, we could easily insist that the overlap of ion tracks is negligible and that NPs are elongated by single impact of SHI. However, $\mathrm{CR}=0.25$ is a somewhat difficult situation to imagine. To understand the geometrical relationship between NPs and tracks more clearly a Monte Carlo computer simulation was carried out.

The size distribution of the Zn NPs before the SHI irradiation was evaluated by SAXS analysis in the previous paper $^{39}$ and was well described in a log-normal distribution of

$$
N(R)=\frac{1}{\sqrt{2 \pi} \ln \beta} \exp \left[-\frac{\left(\ln R-\ln R_{a v}\right)^{2}}{2(\ln \beta)^{2}}\right],
$$

with $R_{a v}=11 \mathrm{~nm}$ and $\beta=1.3$. The SAXS analysis also determined the the mean interparticle distance of $17 \mathrm{~nm}$. For simplicity, monodispersive NPs of $11 \mathrm{~nm}$ in diameter were assumed for the simulation. Since the range of the SHIs was $21.7 \mu \mathrm{m}$, i.e., much longer than the thickness of the layer in which NPs exist, the simulation was simplified to a two-dimmensional one. Assuming a square region of $500 \times 500 \mathrm{~nm}$, NPs of $11 \mathrm{~nm}$ in diameter, i.e., circles of the same diameter, were randomly formed in the region. The total number of the NPs (circles) was set to 1101, in order to meet the experimental value of the mean interparticle distance. After then, ion tracks of $4 \mathrm{~nm}$ in radius were randomly formed in the simulation region. With changing the number of the tracks, i.e., the fluence, the numbers of the tracks which intersected with NP(s) and of the NPs which include overlap of the tracks inside were counted. The simulation was repeated 100 times at each fluence for averaging and the result was shown in Fig. 9.

At the fluence of $5 \times 10^{11} \mathrm{Xe} / \mathrm{cm}^{2}(\mathrm{CR}=0.25), 75 \%$ of NPs were hit once or more by SHIs; i.e., 34\%, 24\%, and $16 \%$ of NPs were hit once, twice, and more than twice, respectively. On average, each NP was affected by 1.4 SHIs. Although $40 \%$ of NPs were hit twice or more, only $6 \%$ of NPs include overlap of tracks inside. Consequently, at $\mathrm{CR}=0.25$, most of NPs were impacted by SHIs but few NPs include track overlap, indicating that the elongation is induced even without the track overlaps.

In contrast, at $2 \times 10^{11} \mathrm{Xe} / \mathrm{cm}^{2}(\mathrm{CR}=0.10), 89 \%$ of NPs were hit only once or less (43\% and $46 \%$ are single-impacted and nonimpacted NPs, respectively). If the elongation was induced at $\mathrm{CR}=0.25$ but not at $\mathrm{CR}=0.10$, we could conclude that the multiple impacts of tracks were essential for the NP elongation. It should be noted that the multiple impacts of NPs by tracks means that different parts of one NP were impacted by different ions, which happens without track overlaps. In fact, only $0.9 \%$ of NPs include the track-overlap inside at $\mathrm{CR}=0.10$. We tried the measurement at $\mathrm{CR}=0.10$ and found 


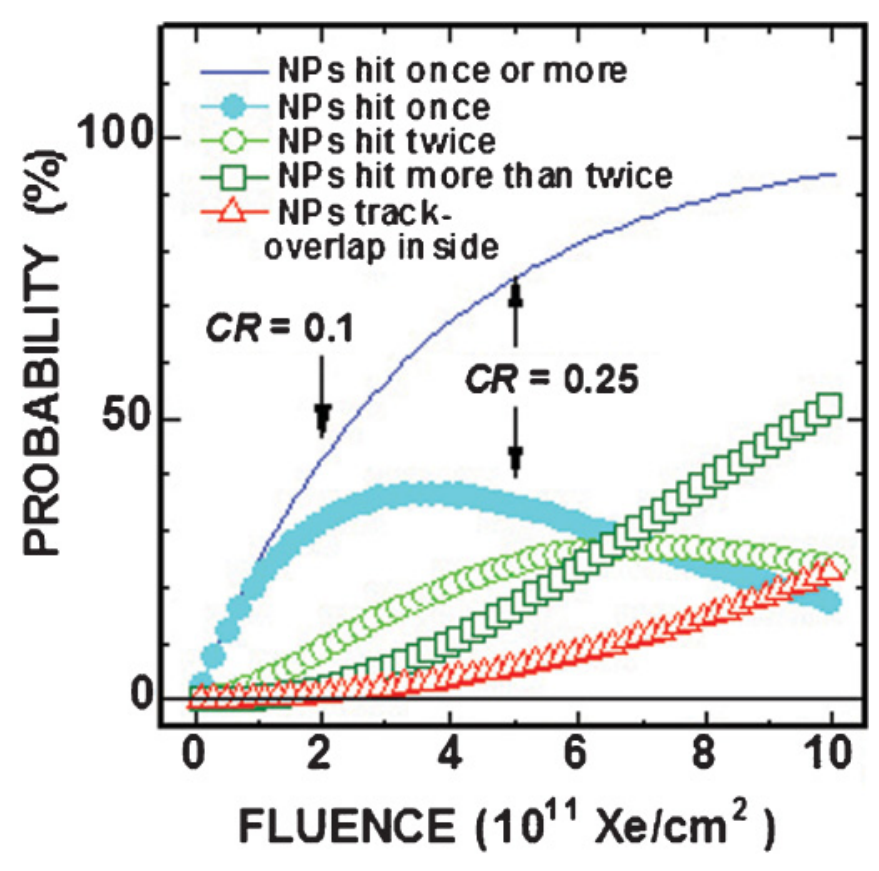

FIG. 9. (Color online) Probabilities of NPs hit by ion tracks simulated by a Monte Carlo method are plotted against the fluence: Those of the NPs hit once or more, only once, twice, and more than twice are indicated by a solid line, solid circles, open circles, and open squares, respectively. Open triangles indicate the probability of NPs which include overlap of ion tracks inside.

signal which was, however, smaller than the experimental uncertainty. The small value is consistent with extrapolation from the data at higher fluences as shown in Fig. 7. At the present we could not judge whether the elongation is induced at $\mathrm{CR}=0.1$.

\section{Comparison with other ion species}

Table II summarized the experimental conditions and degree of the elongation of Zn NPs (this work) and those of Co NPs reported by D'Orleans et al.. ${ }^{7}$ Fortunately both the conditions are very similar: Both used ion energy of $200 \mathrm{MeV}$. Although they used iodine ions $(z=53)$ but we used xenon ions $(z=54)$, iodine and xenon have almost the same atomic numbers. Also the mean diameters of the NPs before the irradiations are 10 and $11 \mathrm{~nm}$, which are very close to each

TABLE II. Comparison of irradiation conditions and aspect ratio (AR) of Co NPs reported by D'Orleans et al. ${ }^{7}$ and those of Zn NPs in this work (m.p., melting point; b.p., boiling point).

\begin{tabular}{lcc}
\hline \hline & D’Orleans et al. & This work \\
\hline NP species & $\mathrm{Co}$ & $\mathrm{Zn}$ \\
Initial mean NP diameter & $10 \mathrm{~nm}$ & $11 \mathrm{~nm}$ \\
m.p. of the bulk state & $1495^{\circ} \mathrm{C}$ & $419.6^{\circ} \mathrm{C}$ \\
b.p. of the bulk state & $2870^{\circ} \mathrm{C}$ & $907^{\circ} \mathrm{C}$ \\
Ion energy for elongation & $200 \mathrm{MeV}$ & $200 \mathrm{MeV}$ \\
Ion species & Iodine $(z=53)$ & Xenon $(z=54)$ \\
AR of NPs at $\sim 5 \times 10^{13}$ ions $/ \mathrm{cm}^{2}$ & $4-5$ & $1.2-1.7$ \\
\hline \hline
\end{tabular}

other. However, ARs of the elongated NPs after the irradiations of $\sim 5 \times 10^{13}$ ions $/ \mathrm{cm}^{2}$ was $4-5$ and $1.2-1.7$ for Co and $\mathrm{Zn}$ NPs, respectively. Although the elongation mechanism has not been clarified yet, some of the major candidate mechanisms suggest the importance of the melting of metal NPs. Since the melting point (m.p.) of $\mathrm{Zn}\left(419.6^{\circ} \mathrm{C}\right)$ is much lower than that of $\mathrm{Co}\left(1495^{\circ} \mathrm{C}\right)$, the smaller AR of $\mathrm{Zn}$ NPs was unexpected.

Awazu et al. calculated time evolution of temperatures of $\mathrm{Au}$ NPs and silica matrix irradiated by SHIs based on the thermal spike model. ${ }^{16}$ According to the calculation of $\mathrm{Au}$ $\mathrm{NP}$ ( $5 \mathrm{~nm}$ in radius) in silica irradiated by $100 \mathrm{MeV} \mathrm{Cu}$ ion, silica starts melting at $5 \times 10^{-14} \mathrm{sec}$ after the ion impact and solidifies at $4 \times 10^{-12} \mathrm{~s}$. However, Au NP start melting at later than $6 \times 10^{-13} \mathrm{~s}$ and solidifies by $3 \times 10^{-11} \mathrm{~s}$. The calculation shows that the silica matrix solidifies first and then NPs do. Since the elongation is expected to be induced only when both the NPs and silica are in molten phases, the moment when the silica matrix solidifies determines the end of the period for the elongation. Even if $\mathrm{Au}$ is substituted by other metal which has much lower m.p., the degree of the elongation is determined mainly by the moment when the silica solidifies. The moment of the solidification does not directly relate with the metal species, although there are many kinds of indirect and (probably) weak influence. Consequently, insensitivity of metal species is expected in the degree of the elongation of NPs: Zn NPs keep the molten phase in silica for a much longer period after the SHI impaction than $\mathrm{Au}$ and Co NPs do. However, most of the molten period does not contribute the elongation since the silica matrix has solidified earlier.

Another peculiarity of $\mathrm{Zn}$ is much lower boiling point (b.p.) of $907^{\circ} \mathrm{C}$, which is lower than the m.p. of Co $1495^{\circ} \mathrm{C}$. Since Co NPs are elongated, i.e., instantaneously melted, under such irradiation conditions, Zn NPs should not only be melted but be simultaneously vaporized. As speculated by D'Orleans et al., the vapor phase does not contribute to the elongation. The low boiling point can be the reason why the elongation of $\mathrm{Zn}$ NPs is not large.

\section{CONCLUSIONS}

Zinc NPs, which were fabricated in $\mathrm{SiO}_{2}$ by implantation of $60 \mathrm{keV} \mathrm{Zn}^{+}$ions, were irradiated by $200 \mathrm{MeV} \mathrm{Xe}^{14+}$ ions at an incident angle of $45^{\circ}$. The fluence of $\mathrm{Xe}$ ions ranged from $1.0 \times 10^{11}$ to $5.0 \times 10^{13} \mathrm{Xe} / \mathrm{cm}^{2}$, which corresponded to $\mathrm{CR}$ values of 0.050 to 25 . It was found from RBS and GIXRD measurements that the total amount of $\mathrm{Zn}$ atoms was almost conserved inside the sample even after the highest fluence of $5.0 \times 10^{13} \mathrm{Xe} / \mathrm{cm}^{2}$ and mainly in the form of crystalline $\mathrm{Zn}$ NPs. However, the GIXRD peak from an amorphous $\mathrm{SiO}_{2}$ matrix steeply decreased with fluence, indicating drastic changes in the structure of the $\mathrm{SiO}_{2}$ matrix. This change was also confirmed as the vibration frequency shifts of the matrix. Both changes saturate around a fluence of $1 \times 10^{13} \mathrm{Xe} / \mathrm{cm}^{2}$, whose dependencies are well fitted by the track coverage using the radius of $4 \mathrm{~nm}$.

The elongation of Zn NPs was monitored by polarized light absorption spectroscopy. A small but measurable dichroism 
was observed at $5.0 \times 10^{11} \mathrm{Xe} / \mathrm{cm}^{2}$, i.e., CR of 0.25 . Supported by the numerical simulation of ion track overlap at this fluence, it was found that the elongation was induced without overlaps of ion tracks.

After further irradiation up to $5.0 \times 10^{13} \mathrm{Xe} / \mathrm{cm}^{2}$, i.e., $\mathrm{CR}$ of 25, each NP experienced multiple interactions with SHIs, which induced further elongation. While the structural changes of the $\mathrm{SiO}_{2}$ matrix saturated around $\sim 1 \times 10^{13} \mathrm{Xe} / \mathrm{cm}^{2}$, the dichroism induced by the elongation increased up to $5.0 \times$ $10^{13} \mathrm{Xe} / \mathrm{cm}^{2}$.

XTEM observations showed that the AR of the elongated NPs ranged from 1.2 to 1.7. However, larger elongation with $\mathrm{AR} \sim 4-5$ was observed in Co NPs at almost the same fluence and irradiation conditions, while the m.p. of $\mathrm{Zn}$ is much lower than that of Co. Furthermore, the b.p. of $\mathrm{Zn}$ is much lower than the m.p. of Co, indicating that the vaporization of Zn NPs might contribute less elongation.

\section{ACKNOWLEDGMENTS}

This work was performed under the Common-Use Facility Program of JAEA. The authors are grateful to the technical staff of the accelerator facilities at JAEA-Tokai for their kind help. A part of this study was supported by "Nanotechnology Network Project" of the Ministry of Education, Culture, Sports, Science and Technology (MEXT), Japan. The authors also thank C. Scholtysik and A. Dahmen (FZ Juelich) for Zn ion implantation, P. Gruber (ETH Zuerich) for helping numerical simulation, and A. Iwase (Osaka Pref. Univ) for stimulating discussion. *amekura.hiroshi@nims.go.jp

${ }^{1}$ R. F. Haglund, L. Yang, R. H. Magruder, C. W. White, R. A. Zuhr, L. Yang, R. Dorsinville, and R. R. Alfano, Nucl. Instrum. Methods B 91, 493 (1994).

${ }^{2}$ U. Kreibig and M. Vollmer, Optical Properties of Metal Clusters (Springer-Verlag, Berlin, 1995).

${ }^{3}$ A. Nakajima, H. Nakao, H. Ueno, T. Futatsugi, and N. Yokoyama, Appl. Phys. Lett. 73, 1071 (1998).

${ }^{4}$ M. Solzi, M. Ghidini, and G. Asti, Magnetic Nanostructures, edited by H. S. Nalwa (American Scientific, Valencia, CA, 2002), Chap. 4, p. 123.

${ }^{5}$ H. Amekura, H. Kitazawa, N. Umeda, Y. Takeda, and N. Kishimoto, Nucl. Instrum. Methods B 222, 114 (2004).

${ }^{6}$ H. Amekura, Y. Fudamoto, Y. Takeda, and N. Kishimoto, Phys. Rev. B 71, 172404 (2005).

${ }^{7}$ C. D'Orleans, J. P. Stoquert, C. Estournes, C. Cerruti, J. J. Grob, J. L. Guille, F. Haas, D. Muller, and M. Richard-Plouet, Phys. Rev. B 67, 220101(R) (2003).

${ }^{8}$ S. Roorda, T. van Dillen, A. Polman, C. Graf, A. van Blaaderen, and B. J. Kooi, Adv. Mater. 16, 235 (2004).

${ }^{9}$ J. M. Lamarre, Z. Yu, C. Harkati, S. Roorda, and L. Martinu, Thin Solid Films 479, 232 (2005).

${ }^{10}$ J. J. Penninkhof, T. van Dillen, S. Roorda, C. Graf, A. van Blaaderen, A. M. Vredenberg, and A. Polman, Nucl. Instrum. Methods B 242, 523 (2006).

${ }^{11}$ A. Oliver, J. A. Reyes-Esqueda, J. C. Cheang-Wong, C. E. RomanVelazquez, A. Crespo-Sosa, L. Rodriguez-Fernandez, J. A. Seman, and C. Noguez, Phys. Rev. B 74, 245425 (2006).

${ }^{12}$ P. Kluth, B. Johannessen, R. Giulian, C. S. Schnohr, G. J. Foran, D. J. Cookson, A. P. Byrne, and M. C. Ridgway, Rad. Effects Defects Solids 162, 501 (2007).

${ }^{13}$ Y. K. Mishra, F. Singh, D. K. Avasthi, J. C. Pivin, D. Malinovska, and E. Pippel, Appl. Phys. Lett. 91, 063103 (2007).

${ }^{14}$ C. Harkati Kerboua, J. M. Lamarre, L. Martinu, and S. Roorda, Nucl. Instrum. Methods B 257, 42 (2007).

${ }^{15}$ R. Giulian, P. Kluth, L. L. Araujo, D. J. Sprouster, A. P. Byrne, D. J. Cookson, and M. C. Ridgway, Phys. Rev. B 78, 125413 (2008)

${ }^{16}$ K. Awazu, X. Wang, M. Fujimaki, J. Tominaga, H. Aiba, Y. Ohki, and T. Komatsubara, Phys. Rev. B 78, 054102 (2008).
${ }^{17}$ P. Kluth, C. S. Schnohr, O. H. Pakarinen, F. Djurabekova, D. J. Sprouster, R. Giulian, M. C. Ridgway, A. P. Byrne, C. Trautmann, D. J. Cookson, K. Nordlund, and M. Toulemonde, Phys. Rev. Lett. 101, 175503 (2008).

${ }^{18}$ J. A. Reyes-Esqueda, C. Torres-Torres, J. C. Cheang-Wong, A. Crespo-Sosa, L. Rodriguez-Fernandez, C. Noguez, and A. Oliver, Opt. Express 16, 710 (2008).

${ }^{19}$ M. C. Ridgway, P. Kluth, R. Giulian, D. J. Sprouster, L. L. Araujo, C. S. Schnohr, D.J. Llewellyn, A. P. Byrne, G. J. Foran, and D. J. Cookson, Nucl. Instrum. Methods B 267, 931 (2009).

${ }^{20}$ S. Klaumunzer, Nucl. Instrum. Methods B 244, 1 (2006).

${ }^{21}$ E. A. Dawi, G. Rizza, M. P. Mink, A. M. Vredenberg, and F. Habraken, J. Appl. Phys. 105, 074305 (2009).

${ }^{22}$ B. Schmidt, K. H. Heinig, A. Mueklich, and C. Akhmadaliev, Nucl. Instrum. Methods B 267, 1345 (2009).

${ }^{23}$ T. van Dillen, A. Polman, C. M. van Kats, and A. van Blaaderen, Appl. Phys. Lett. 83, 4315 (2003).

${ }^{24}$ H. Amekura, H. S. Wang, S. Hishita, J. Pan, N. Kishimoto, C. Buchal, and S. Mantl, Nanotechnology 20, 065303 (2009).

${ }^{25}$ G. Rizza, E. A. Dawi, A. M. Vredenberg, and I. Monnet, Appl. Phys. Lett. 95, 043105 (2009).

${ }^{26}$ H. Amekura, M. Tanaka, Y. Katsuya, H. Yoshikawa, H. Shinotsuka, S. Tanuma, M. Ohnuma, Y. Matsushita, K. Kobayashi, C. Buchal, S. Mantl, and N. Kishimoto, Appl. Phys. Lett. 96, 023110 (2010).

${ }^{27}$ H. Amekura, M. Tanaka, Y. Katsuya, H. Yoshikawa, M. Ohnuma, Y. Matsushita, K. Kobayashi, and N. Kishimoto, J. Appl. Phys. 108, 104302 (2010)

${ }^{28}$ H. Amekura, N. Umeda, K. Kono, Y. Takeda, N. Kishimoto, C. Buchal, and S. Mantl, Nanotechnology 18, 395707 (2007).

${ }^{29}$ H. Amekura, N. Umeda, Y. Sakuma, N. Kishimoto, and C. Buchal, Appl. Phys. Lett. 87, 013109 (2005).

${ }^{30}$ H. Amekura, N. Umeda, Y. Sakuma, O. A. Plaksin, Y. Takeda, N. Kishimoto, and C. Buchal, Appl. Phys. Lett. 88, 153119 (2006).

${ }^{31} \mathrm{H}$. Amekura and N. Kishimoto, in Lecture Notes in Nanoscale Science and Technology, edited by Z. Wang (Springer, New York, 2009), Vol. 5, pp. 1-75. 
${ }^{32}$ J. F. Ziegler, J. P. Biersack, and U. Littmark, The Stopping and Range of Ions in Solids (Pergamon, New York, 1985).

${ }^{33}$ L. R. Doolittle, Nucl. Instrum. Methods B 15, 227 (1986).

${ }^{34}$ B. Joseph, J. Ghatak, H. P. Lenka, P. K. Kuiri, G. Sahu, N. C. Mishra, and D. P. Mahapatra, Nucl. Instrum. Methods B 256, 659 (2007).

${ }^{35}$ B. Johannessen, P. Kluth, D. J. Llewellyn, G. J. Foran, D. J. Cookson, and M. C. Ridgway, Phys. Rev. B 76, 184203 (2007).
${ }^{36}$ D. J. Sprouster, R. Giulian, L. L. Araujo, P. Kluth, B. Johannessen, N. Kirby, K. Nordlund, and M. C. Ridgway, Phys. Rev. B 81, 155414 (2010).

${ }^{37}$ R.A. B. Devine, J. Non-Cryst. Solids 152, 50 (1993).

${ }^{38}$ C. F. Bohren and B. R. Huffman, Absorption and Scattering of Light by Small Particles (John Wiley \& Sons, New York, 1983).

${ }^{39}$ H. Amekura, M. Ohnuma, N. Kishimoto, C. Buchal, and S. Mantl, J. Appl. Phys. 104, 114309 (2008). 